\title{
Medical care and clinical practice for disabled elderly
}

Rui-Fang Zhua,b, Shi-Fan Han ${ }^{\mathrm{b}, *}$

aSchool of Nursing, Shanxi Medical University, Taiyuan, Shanxi 030001, China

${ }^{b}$ First Hospital of Shanxi Medical University, Taiyuan, Shanxi 030001, China

Received: 12 July 2017; Accepted: 27 November 2017; Published: 20 March 2018

Abstract: At present, with the continuous development of the trend of population aging worldwide, more elderly people are increasingly facing serious life disorders caused by physical and mental disability. In this study, the common symptoms and the nursing practice related to diseases of the disabled elderly are reviewed with reference to the basic concepts of disability care and the current situation of domestic and foreign research, which is expected to provide the basis for the construction of a new model of disability nursing.

Keywords: disability $\bullet$ elderly $\bullet$ disability care $\bullet$ clinical practice $\bullet$ symptoms $\bullet$ disease

(c) Shanxi Medical Periodical Press.

\section{Introduction}

The life of every elderly individual carries profound memories. They meticulously raised their children; they put all their efforts, without any hesitation, into helping their children purchase a house, get married, and have children, as well as into taking care of the grandchildren. Bearing in mind the growing trend of population aging in China and the various difficulties of the disabled elderly, we should seriously consider what we can do for the elderly. Elderly disability is not only a natural phenomenon but also a social phenomenon. It is extremely important to provide professional care services for the disabled elderly.

\section{The concept of disability care}

The World Health Organization (WHO) defines people older than 65 years of age in developed countries and people older than 60 years of age in developing countries as the elderly. In China, the Geriatrics Society of the Chinese Medical Association recommends that people older than 60 years of age be defined as elderly. ${ }^{1}$ China entered an aging society in 2000. According to the "Chinese report of the development of a livable environment for the elderly (2015)", released by the China Research Center on Aging in 2016, China's advanced elderly population has reached 25 million, and the population of disabled elderly has surpassed 40 million.

Disability refers to the loss of life or social ability due to accidental injury or illness leading to physical or mental damage. The purpose of disability care is to investigate, diagnose, and treat the response of the disabled elderly to their own existing and potential health problems, with the disabled elderly as the research object, thereby protecting, promoting, and optimizing the health and viability of the elderly. In layman's terms, disability care is the provision of continual, long-term, and comprehensive care services 
in medicine, nursing, and living for the elderly who have difficulty in taking care of themselves. This comprehensive care service includes both daily life care and professional care in all aspects of prevention, treatment, rehabilitation, and other features. Especially for elderly individuals who have underlying diseases, the development of a comprehensive, personalized, and professional care program is particularly important for the protection of their most basic quality of life.

\section{Overview of disability care in China and other countries}

In the United States, apartments for senior citizens with assistants are provided for the partially disabled elderly, in which mostly elderly individuals older than 65 years of age reside and the necessary assistance for daily life is provided; senior citizens' apartments with continuing care (special care) are provided for the disabled elderly to facilitate health care services, including care and rehabilitation treatment services by professional nurses and health monitoring services.

In Germany, medical care for the disabled elderly mainly includes four basic services: the first is personal hygiene services for the disabled elderly, including combing their hair, brushing their teeth, and bathing. The second is nutrition services, i.e., preparing meals and helping the disabled elderly eat. The third is the provision of services for the disabled elderly in daily activities, such as helping them go outdoors, get out of bed, walk, and step up and down stairs. The fourth is housekeeping services, i.e., helping the disabled elderly in laundry, cooking, and shopping, among others.

In Sweden, the government has built apartments for the elderly in ordinary residential areas, thus allowing the partially disabled elderly to live near their children; alternatively, it has constructed units with assistance facilities for the disabled elderly in residential buildings based on their conditions. In general, the elderly people living in nursing homes are lonely elderly with a serious loss of self-care ability.

In Australia, the Red Cross has a special care program for the disabled elderly. For care receivers older than 65 years of age, a personalized intensive care program is provided, and respite care services help senior care receivers maintain their status.

In Japan, many companies have built commercial nursing homes with personalized care services for the elderly with physical inconvenience and diseases, and care services for the disabled elderly are provided by an affiliate team of the nursing home. In Japan, nursing is one of the essential skills of life, similar to qualifying computer level two or having a driver's license. The Japanese believe that nursing skills can not only help them provide services for their family but also play a decisive role at crucial moments.

In Singapore, community hospitals, structured in combination with medicine and pension provision, for the disabled elderly have been established to provide the corresponding nursing services and rehabilitation treatment. For low-income and poor elderly with disability and partial disability, the opportunity to reside in volunteer welfare agencies is provided. When a disabled elderly individual is diagnosed with a fatal disease by a professional diagnosis and treatment institution, psychological support for the elderly individual is also provided by hospice care institutions for the disabled elderly.

In China, there has been a concept that raising children is for those in old age. Before the modern era, caring for the disabled elderly mainly consisted of home care. Recently, care for the disabled elderly has mainly included the family-based model, the institution-based model, and the community home-based model. In recent years, the size of the family has gradually tended to be miniaturized, the number of dual-career families has gradually increased, and the probability of and the time for caring for the disabled elderly have shown a substantial increase, and the requirements of the disabled elderly in terms of quality of life and care have also risen. However, the development of disability care remains far from meeting the needs of the elderly. The increase in the elderly population has greatly increased the burden on the community and on families. In particular, the demand for medical care services by empty nesters and the sick elderly of advanced age has increased rapidly. Therefore, we should learn from the foreign advanced experience and, in accordance with our own national conditions, develop our own pension model and actively promote the development of China's elderly care facilities or services.

\section{Management of disability nursing staff}

\subsection{Ethical principles in disability nursing}

In the process of long-term care for the elderly by nursing staff, the assimilation of the elderly, unexpected events, breaches of contract, disclosures of confidential information, accidents, mistakes, and disputes may occur. In this case, the nursing staff should respect every elderly individual so as to avoid potential harm or 
reduce the actual harm done, develop their own spirit of caution, treat everyone fairly, and fulfill their duty in strict accordance with operating rules and ethical norms.

(1) The principle of respect: in care services for the disabled elderly, the rights of informed consent for the elderly, the autonomy of the elderly, and the privacy of the elderly should be respected.

(2) The principle of no harm: this principle involves not harming the disabled elderly in the process of care, including physical damage (such as pain, complications, injuries, disability, and death), and mental and social damage (such as mental suffering, economic loss, and being insulted and discriminated against).

(3) The principle of favorableness: according to this principle, caregivers should behave in a manner that is beneficial to the elderly. In the nursing process, the risk-benefit ratio must also be evaluated to fully consider behaviors that have more advantages than disadvantages.

(4) The principle of impartiality: this principle entails no partiality, an unbiased attitude, and integrity. It is mainly reflected in the personal behavior of the nursing staff, which should be strictly regulated in a fair and honest manner.

\subsection{Self-management by disability nursing staff}

The main task of the nursing staff is to move the elderly. This action may be repetitive in the nursing process, with a certain difficulty that requires great physical strength on the part of the caregiver. If the movement is not smooth enough, the caregiver can easily become injured, which may also lead to the discomfort of the disabled elderly. Therefore, mastering the appropriate nursing skills is very helpful in nursing services.

(1) Mastering the conditioning method by body posture: an appropriate posture can effectively reduce the burden of the caregiver and the patient. Therefore, full consideration should be given to the standing position, the location of the objects, and adjusting for the height of the bed, among other matters, in caring for the disabled elderly.

(2) Establishing good living habits: caregivers should maintain a regular and balanced diet and exercise routinely, with a relaxed mood, to enhance their physical strength; allocate enough time to take a bath and relieve fatigue; and ensure an adequate amount of time for sleep. ${ }^{2}$

\section{Care for the disabled elderly}

\subsection{Care for the disabled elderly with common symptoms}

Aging and chronic diseases cause a variety of symptoms and health problems in the skin and in the nervous, cardiovascular, respiratory, urinary, digestive, and musculoskeletal systems of the disabled elderly. For these specific health problems, appropriate care services can improve the self-care ability of the disabled elderly and prevent the occurrence of new diseases.

\subsubsection{Pressure sore}

A pressure sore is tissue damage and necrosis due to long-term pressure on local tissue, blood circulation disorders, sustained ischemia and hypoxia in local tissue, and nutritional deficiencies, resulting in the loss of normal function of the skin. Long-term bedridden elderly or elderly individuals having difficulty in body movement are prone to the most serious skin problems, characterized by high incidence, rapid development of disease, difficult cure, and recurrence after healing. Once a pressure sore occurs, it not only causes discomfort to the elderly, aggravates the disease condition, and prolongs the rehabilitation of the disease but also poses a threat to the patient's life if sepsis occurs due to a secondary infection in severe cases. Therefore, we must pay full attention to skin care for the disabled elderly to prevent and reduce the occurrence of pressure sores. ${ }^{3}$

\subsubsection{Case analysis}

A patient, Mr. Tang, male, 79 years old, was diagnosed with cerebral infarction, diabetes mellitus (DM), laryngeal cancer, and multiple organ dysfunction syndrome. The patient was in clouded consciousness on admission, showing erythema on the back of both the lower limbs, which did not fade when pressure was applied, occasional pain, and movement disorders on the righthand side of the body after the cerebral infarction. After admission, ventilator-assisted breathing and anti-shock treatments were provided.

\subsubsection{Care}

The patient had a stage I pressure sore, i.e., the stage with congestion and redness; stages II, III, and IV are the stages of inflammatory infiltration, shallow ulcer, and necrotic ulcer, respectively. Now, how are pressure sores prevented and cared for? (1) Long-term local tissue pres- 
sure should be avoided: the body of the patient should be flipped every 2 hours, and actions of dragging, drawing, and pulling should be avoided while flipping. If necessary, the interval between changing the patient's body posture can be shortened, with the protection of the bone bulge occurring simultaneously. Support for the body gap, protective equipment, and decompression equipment can be used. It is best to use a medical electric anti-bedsore mat, if possible. (2) The stimulation of moisture, friction, and excreta should be avoided: the bed should be clean, dry, and smooth without debris. The elevation of the head of the bed should not exceed $30^{\circ}$. If necessary, a lifting device by hanging can be used. The semirecumbent posture should be correct. (3) Manual massage should be provided in some situations: the thenar and hypothenar eminences of both hands are dipped in a little $50 \%$ ethanol to perform a centripetal massage or a ring-shaped outward massage with the thumb pulp for 3-5 minutes, which should be repeated numerous times. The order of the massage is as follows: the top of the hip $\rightarrow$ upward massage along the spine $\rightarrow$ shoulder $\rightarrow$ turning down to the waist (hypothenar eminences for upward massage and thenar eminences for downward massage), twice; the sacrococcygeal region is massaged using the palms (between the thenar eminences) for 3 minutes; and start from the sacrococcygeal region $\rightarrow$ massage along the spine to the seventh cervical spine. The massage should be performed using the thumb pulp twice, with talcum powder applied evenly on the back.

\subsubsection{Edema}

Edema is the accumulation of excessive fluid in the tissue gap. Edema can be divided into recessive edema and dominant edema, and it can also be divided into systemic edema and local edema. Common types of local edema include inflammatory edema, venous obstructive edema, and lymphedema. Other types of edema include cardiac edema (the edema first occurs in the lower limbs), renal edema (edema at the eyelids and face in morning), and liver edema (liver palm and spider nevus). When an edema occurs in the elderly, swelling, lighter wrinkles, and declined elasticity of the skin are observed, as well as a depression on the skin when pressed with a finger. Long-term continuous edema can cause skin damage and secondary infection, and in severe cases, the water-sodium retention may also cause an increase in blood volume, an increase in cardiac load, or even heart failure.

\subsubsection{Case analysis}

A patient, Mrs. Feng, female, 63 years old, had suffered from hemiplegia for 3 years and was admitted due to hypertension for 15 years, right-sided heart failure for 1 month, and lumbosacral and medial thigh edema for 2 weeks.

\subsubsection{Care}

This patient was diagnosed with cardiac edema. For the long-term bedridden elderly, cardiac edema is most obvious at the lumbosacral and medial thigh region, which gradually spreads to the entire body; the edema in the early stage may show significant changes between day and night, with the most severe edema occurring before bedtime; in the advanced stage, the edema is systemic, and in general, the face is not swollen. For the elderly with edema, the skin of the edema should be well protected; activities should be limited with more bed rest; a low-salt diet should be provided, with 2-3 g/day being appropriate. The water intake of patients with cardiac edema is generally not limited, but the elderly with renal edema should not drink excessive water. If the daily urine output is $<500 \mathrm{~mL}$, then the intake of liquid should be restricted, and the water intake for severe cases should be based on the output of urine. The elderly with edema in their lower extremities should reduce their standing or sitting time and attempt to lie flat if possible, with the lower limbs raised to reduce the edema; the elderly with scrotal edema should lift the scrotum using a scrotum holder or soft towel to assist the subsiding of the edema, and skin ulceration should be carefully prevented.

\subsubsection{Paralysis}

Paralysis is the reduction or loss of free movement function. The former concerns mild paralysis or incomplete paralysis, whereas the latter concerns complete paralysis. Paralysis can have different ranges, including single paralysis, hemiplegia, crossed paralysis, paraplegia, and quadriplegia. The paralyzed elderly cannot maintain a normal position and must lie down or sit (in a wheelchair) for long periods of time, which limits or reduces their activities and may lead to a variety of systemic complications, such as pressure sores, partial muscle atrophy, orthostatic hypotension, deep vein thrombosis, hypostatic pneumonia, malnutrition, excretion difficulty, and even psychological problems such as anxiety and fear.

\subsubsection{Case analysis}

A patient, Mr. Yang, male, 68 years old, could not take care of himself in daily life due to a sudden cerebral infarction and hemiplegia 1 year earlier, with side leakage while eating and unconscious drooling. 


\subsubsection{Care}

This patient is a senior citizen with hemiplegia, and complications - including pressure sores, lung infection, constipation, and dysuria - should actively be prevented during care. A comfortable and relaxed body posture should be maintained. When conditions allow, the body position should be frequently changed, or activities such as walking should be performed. Safety should be a concern, and accessible facilities should be set up.

The functional position of each joint should be maintained as much as possible to prevent joint contracture and loss of function. (1) The paralyzed body can be supported using soft pillows, and the palm can hold a small scroll to maintain the extension of the knuckles; in the supine position, a soft pillow can be placed in the hip joint to prevent the external rotation of the hip; the ankle can be fixed with a foot holder to prevent foot sagging; the paralyzed upper limb can be hung while sitting or standing to prevent shoulder dislocation. (2) It is easy for paralyzed muscles to become cold due to a blood circulation disorder; thus, the body temperature of the patient must be maintained as warm, particularly in the case of the lower limbs; wearing socks is recommended, but not the use of hot water for heating so as to prevent burns. The paralyzed limbs can be massaged to promote blood circulation and prevent muscle atrophy.

Joint movements, in full range, should be performed to maintain the mobility of the joints: for the elderly with limited physical activity, passive activities can be used in exercises to encourage the elderly to cooperate, thus allowing the joints and muscles to obtain the maximum range of exercise. For the elderly who can get out of bed for activities, active activities can be used to complete the isometric exercise and isotonic exercise of the muscles by hand or with simple devices.

\subsubsection{Pain}

Pain is an unpleasant subjective and emotional experience associated with tissue injury or potential tissue injury. The incidence of chronic pain in the elderly is $25 \%-50 \%$, and $45 \%-80 \%$ of patients suffering from chronic pain require long-term treatment and care; $>70 \%$ of elderly patients with tumors showed associated pain symptoms, which may be complicated by depression, sleeping disorder, decreased joint activity, and physical and social cognitive disorders. In other words, pain may not only originate from the tumor, but sometimes, it may also be accompanied by pain caused by other diseases such as arthritis, osteoporosis, and DM. For patients suffering from pain, relieving the pain is an active treatment, and controlling the pain is a key factor to improve their quality of life. ${ }^{4}$ It is therefore important to accurately assess it and to take effective care measures to ease the pain.

\subsubsection{Case analysis}

A patient, Mr. Cai, male, 70 years old, sought treatment in Lvliang People's hospital due to "a choking feeling when swallowing". Recently, the patient was admitted because of "a left ear edema for 1 year and left upper chest pain for 2 months", with the diagnosis of "esophageal squamous cell carcinoma at the upper thorax segment, with metastasis of the bilateral supraclavicular lymph nodes" and "cancer pain".

\subsubsection{Care}

For patients suffering from cancer pain, caregivers should provide a targeted, comprehensive intervention program that includes psychological counseling, health education, and drug analgesia. In general, according to the "three levels" analgesic program recommended by the $\mathrm{WHO}$, analgesic drugs and adjuvant drugs for the corresponding level should be given, following the principles of oral administration, on-time administration, corresponding level administration, individualized dosage, and attention to specific details. In the process of nursing, observing and addressing the side effects of drugs are the most important issues, particularly the prevention of constipation because the incidence of constipation is as high as $90 \%-100 \%$. Additionally, the occurrence of nausea, vomiting, and lethargy, as well as excessive sedation, in patients should be carefully observed. Occasionally, muscle relaxation training can be performed so that patients can consciously control their own physiological and psychological activities, thus reducing the level of awakening and improving physical and psychological disorders, to achieve the purpose of treatment and care.

\subsubsection{Pipeline slippage}

Because of the disease conditions, a variety of indwelling pipelines are often required for the disabled elderly. These pipelines have different functions, often as a means of treatment and for observation of the disease, as well as forming the basis of prognosis. They are directly related to the outcome of the patient's disease and life safety. Thus, they are very important. For example, supply pipelines include the oxygen tube, the nasogastric feeding tube, the gastrointestinal nutrition tube, the infusion tube (including the subclavian vein puncture catheter, the catheter of the central vein with 
peripheral placement, and the indwelling catheter in other parts of the body). Discharge pipelines include the gastrointestinal decompression tube, the bladder fistula, the indwelling catheter, the ventricle drainage tube, the closed thoracic drainage tube, and the "T" tube. Monitoring pipelines include the central venous pressure tube and the electrocardiogram (ECG) monitoring tube.

\subsubsection{Case analysis}

A patient, Mr. Zhao, male, 68 years old, was admitted due to "progressive dysuria for six 6 years" as and "prostate hyperplasia". The patient showed disability with a history of chronic bronchitis, hypertension, and coronary heart disease. Under continuous epidural anesthesia, after the transurethral resection of the prostate, catheterization was performed in the deep vein at the right neck for infusion, and a three-chamber catheter was placed within for continuous bladder rinse. Vital signs were monitored as being stable.

\subsubsection{Care}

This is a patient who needed the pipeline care. First, smooth drainage should be maintained. The drainage catheter should be routinely checked to avoid twisting, folding, displacement, compression, fall-off, and blockage. If drainage is not smooth, with suspected clogging, then the drainage tube can be squeezed from the proximal end to the distal end; if the drainage tube is certainly blocked, then the drainage tube should be squeezed or rinsed with sterile isotonic saline. Note that the principles of aseptic operation should be strictly followed. Second, the pipelines should be properly fixed. The drainage tube should be properly fixed by the bed of the elderly patient, with particular attention to the fixation of the joints, to prevent falling off, and the in vitro part can be fixed using safety pins to prevent it from being unintentionally unplugged by the elderly patient before waking up from anesthesia. In addition, the length of the drainage tube should be appropriate. If it is too long, then it will be easy for it to distort and increase the dead space, thus affecting drainage; if it is too short, then it will not be conducive to the activities of the elderly patient, such as flipping in the bed and getting out of the bed. Elderly patients should try to wear clothes with a front opening and be careful not to take out the tubing when putting clothes on, taking clothes off, or changing clothes. For the elderly with an irritability problem and consciousness disorder, multidirectional fixation should be performed. Additionally, the drainage tube should always be placed lower than the outlet of the drainage tube to avoid the backflow of the draining fluid, causing a retrograde infection in the elderly. In the case of closed-chest drainage, the liquid level of the water-sealing bottle is generally $60 \mathrm{~cm}$ lower than the chest outlet of the drainage tube. For drainage with a "T"-shaped tube, the drainage bag should not be placed too low to avoid excessive bile loss. ${ }^{5}$

\subsection{Care for the common diseases of the disabled elderly}

One of the characteristics of the disabled population in China is the diseases. These elderly people have lost the ability of self-care due to illness, and disability also brings on more chronic diseases. According to statistics, the prevalence of chronic diseases in the elderly in all regions was $>90 \%$, with the proportion of disability caused by the disease sequelae accounting for $>70 \%$. Among the diseases that increase the risk of disability in the elderly, hypertension, cerebral apoplexy (commonly known as "stroke"), and DM are common.

\subsubsection{Chronic obstructive pulmonary disease in the elderly}

Chronic obstructive pulmonary disease (COPD) in the elderly involves a group of lung diseases caused by chronic airway obstruction and characterized by ventilation dysfunction, and the airflow limitation is not completely reversible, showing progressive development. However, COPD can be prevented and treated. It mainly includes chronic bronchitis and obstructive pulmonary emphysema, which is a common and frequently occurring disease in the elderly. With the increase in age, the incidence increases, and it is currently ranked as the fourth leading cause of death worldwide.

\subsubsection{Case analysis}

A patient, Mr. Zhang, male, 75 years old, sought treatment 3 days ago due to fever, severe cough, with a considerable amount of thick yellow sputum, shortness of breath, and cyanosis caused by a cold. The patient had a more than 30-year history of smoking and a more than 20-year history of chronic cough with sputum. The physical examination revealed the following: temperature: $39.2^{\circ} \mathrm{C}$; white blood cell count: $13.0 \times 10^{9} / \mathrm{L}$; and arterial carbon dioxide partial pressure: $65 \mathrm{mmHg}$.

\subsubsection{Care}

(1) Life guidance: guide patients to avoid becoming cold; to avoid contact with patients with respiratory infections; to avoid inhalation of dust and irritating gas; 
to have a high-calorie, high-protein, high-vitamin, and digestible diet; to avoid food that causes gas or constipation; and to drink plenty of water. (2) Oxygen therapy guidance: in general, a nasal catheter is used to provide a continuous low flow of oxygen, with a flow of $1-2 \mathrm{~L} / \mathrm{min}$, a concentration $<30 \%$, and daily humidified oxygen inhalation for 15 hours or more. (3) Deep breathing and effective cough: instruct patients to perform several free breaths every $2-4$ hours, with a strong cough after holding a moment at the end of an inhalation, to promote the movement of secretions from the distal airway toward the main airway with the air flow. (4) Chest tapping: by tapping the back with vibration, the sputum attached to the alveolar and bronchial wall can indirectly be loosened off. The fingers should be close together and slightly bending toward the palm, forming an empty palm. The wrist is relaxed to quickly and regularly tap the chest. The tapping is from bottom to top and from outside to inside, for 15-20 minutes, and 2-3 times a day before meals. During the tapping, the skin color, breathing, coughing, and expectoration should be carefully observed, and the tapping should immediately be stopped if the patient feels uncomfortable. It is better to use mechanical sputum-discharge assist device, if possible. (5) Drainage by body position: with the opening of the lesion downward, gravity, effective coughing, or chest tapping is used to excrete the secretions. Drainage is often performed in 1 hour before breakfast, before dinner, and before bedtime, for 10-15 minutes each time. (6) Breaths with puckered lips: to inhale using the nose and exhale using the mouth. While exhaling, the lips can pucker into the shape for blowing a flute, and the gas is slowly exhaled through the narrowed lips. The ratio of inhalation and exhalation is $1: 2$ or $1: 3$, for $10-20$ minutes each time and twice a day, and it is expected to become a natural breathing habit. (7) Abdominal breathing: the patient is in a sitting or standing position, with one hand on the abdomen and the other hand on the chest. In inhalation, the abdomen is pushed up as far as possible without moving the chest; in exhalation, the abdomen is retracted to exhale the gas as far as possible, 7-8 cycles every minute, 10-20 minutes each time, and twice a day. A variety of breathing exercises can also be applied to patients in severe conditions or to those who are not willing to participate in the above respiratory muscle exercise.

\subsubsection{Elderly hypertension}

Hypertension is the clinical manifestation mainly manifested as continuously rising arterial systolic blood pressure (SBP) and/or diastolic blood pressure (DBP), which is one of the clinical syndromes with the high- est incidence. Elderly hypertension involves patients older than 60 years of age who show three consecutive occurrences of average systolic blood pressure (SBP) $\geq 140 \mathrm{mmHg}$ and/or diastolic blood pressure (DBP) $\geq 90$ $\mathrm{mmHg}$ measured on different days with no drug treatment, excluding pseudo-hypertension and secondary hypertension. Elderly hypertension is the most common disease in the elderly, with an incidence rate of $40 \%$ (38\% in men and $42 \%$ in women), and it is the main cause of death and disability in the elderly.

\subsubsection{Case analysis}

A patient, Mr. Zhang, male, 75 years old, complained of chest pain lasting 20 minutes at 6 am while sleeping and was sent to the emergency room accompanied by family members, showing an SBP of $160 \mathrm{mmHg}$ and a DBP of $110 \mathrm{mmHg}$. He was admitted with "hypertension and acute coronary syndrome, with unstable angina". The patient had an 8-year history of hypertension, with intermittent administration of antihypertensive drugs, and his BP control was poor.

\subsubsection{Care}

(1) Life guidance: The patient should take bed rest, with limited sodium intake and increased crude fiber in his diet to prevent constipation. (2) BP monitoring: the BP should be measured three times a day, with the "four fixed factors" (fixed time, fixed part [right arm or left arm], fixed posture [lying or sitting], and fixed sphygmomanometer) as much as possible. (3) Medication care: the importance of long-term drug treatment is emphasized. Patients should not increase or decrease, or even suddenly stop, drug administration by themselves. (4) Nitroglycerin: nitroglycerin is the most effective common medicine for relieving angina. Before the oral administration of nitroglycerin, the mouth should be moisturized with water, and the drug is then chewed and placed under the tongue, which is conducive to the rapid melting of the drug for functioning. If conditions allow, a nitroglycerin spray can also be used. It should be noted that the elderly should lie down flat when using this drug for the first time.

\subsubsection{Acute myocardial infarction in the elderly}

Acute myocardial infarction in the elderly may involve an intracoronary plaque rupture owing to coronary artery disease, forming a thrombosis or coronary artery spasm that is severe and persistent; this, in turn, causes acute coronary artery occlusion and a rapid decrease or interruption of the coronary blood supply, leading to 
a continuous and severe ischemia of the corresponding myocardium, resulting in myocardial necrosis. Its clinical manifestations include persistent severe pain behind the sternum, fever, increased levels of white blood cells and serum myocardial necrosis markers, and progressive changes in ECGs. It is often complicated by acute circulatory failure and severe arrhythmia. The incidence of acute myocardial infarction in the elderly is significantly higher than that in young and middle-aged people. With increase in age, the incidence gradually increases, with a high mortality rate.

\subsubsection{Case analysis}

A patient, Mr. Wang, male, 63 years old, suffered from repeated chest tightness and chest pain for 6 years and was admitted half an hour after aggravation. The diagnosis on admission was "acute extensive anterior myocardial infarction with ST segment elevation". The physical examination showed the following: temperature: $38.2^{\circ} \mathrm{C}$; increased white blood cells; and an increased erythrocyte sedimentation rate. The peaks of creatine kinase (CK), aspartate transaminase (AST), and lactate dehydrogenase (LDH) were delayed. The durations of the CK and AST peaks were long. The CK peak value was low.

\subsubsection{Care}

(1) Life guidance: Within 4-12 hours after onset, the patient should be given a fluid diet to reduce gastric dilatation, followed by the transition to a light diet, with a small amount for each meal. Within 12 hours of onset, the patient needs absolute bed rest in a quiet environment. (2) Thrombolysis guidance: during the thrombolysis, the patient should be asked about his or her history of cerebrovascular disease and active bleeding, as well as the bleeding tendency; adverse reactions to thrombolytic drugs, such as allergic reactions, hypotension, and bleeding, should be carefully observed. The ECG and BP should be closely monitored. (3) Drug treatment: morphine (analgesic) has the side effects of inhibiting respiration and lowering the $\mathrm{BP}$ and heart rate. The tolerance of elderly patients to morphine is reduced; thus, adverse reactions such as respiratory depression should be closely observed during the use of morphine. For patients with obstructive pulmonary emphysema and other lung diseases, the use of this drug is not allowed. Anticoagulants can prevent early infarct expansion, thus preventing the extension of coronary thrombosis to the proximal segments. Gastrointestinal reactions and the bleeding condition should be carefully observed when using these drugs.
Angiotensin-converting enzyme inhibitor can reduce secondary myocardial infarction and improve the longterm survival rate. However, it has the side effects of causing hypotension, dizziness, fatigue, renal dysfunction, cough, and angioedema; thus, the BP, serum potassium concentration, and renal function should be closely monitored in the medication process.

\subsubsection{DM in the elderly}

DM comprises a group of chronic metabolic diseases characterized by an increased blood glucose level, which is caused by a relative or absolute deficiency of insulin secretion and/or a defect in its function, and it is one of the most common diseases of the endocrine system. With increase in age, the incidence of DM shows a clear upward trend. In elderly people, type $2 \mathrm{DM}$ is more common, and patients are typically overweight. Common symptoms and signs include the following: metabolic disorders syndrome: "three high and one low", i.e., "increased thirst, hunger, and frequent urination, as well as weight loss"; ketoacidosis: only polyuria, polydipsia, and fatigue occur in the early stage, followed by loss of appetite, nausea, vomiting, headache, lethargy, and deep breathing with a rotten apple smell; dehydration: this complication is obvious in the late stage, showing oliguria, dry skin, lowered BP, shock, coma, and even death; and hypoglycemia: the self-healthcare capacity and compliance of the elderly are poor, resulting in poor blood glucose control. In the case of improper medication, the occurrence of hypoglycemia is frequently the result. At present, DM and its complications have become the main cause of death of the elderly after cardiovascular disease; thus, we must pay attention to them.

\subsubsection{Case analysis}

A patient, Mrs. Li, female, 62 years old, was diagnosed with DM during a physical examination 3 years earlier. The patient was administered irregular oral hypoglycemic agents, with blood sugar fluctuating at approximately $10 \mathrm{mmol} / \mathrm{L}$, urine sugar of $(+)-(+++)$, and BP of $150 / 100 \mathrm{mmHg}$. Recently, she complained about chest tightness, dizziness, and fatigue.

\subsubsection{Care}

(1) Diet control: the diet for the patient should be provided in strict accordance with the planned amount and the planned time. Food with a low sugar content and rich in cellulose and vitamins, which are thirst-quenching and provide a feeling of satiety, is recommended, and the salt 
intake should be well controlled. (2) Exercise therapy: exercises that the elderly can handle, such as walking, light gymnastics, and tai chi, are recommended. They should do exercises step by step, with perseverance, and in a regular daily load to avoid excessive fatigue. The exercise time should range from 30 minutes to 1 hour after meals, and exercise with fasting should be avoided. In addition, when exercising, particularly during outdoor exercise, snacks or food should be prepared to avoid hypoglycemia. (3) Blood glucose monitoring: monitoring blood glucose is very important for patients with DM. It can be regularly monitored at home using a test paper or a blood glucose monitor. (4) Drug treatment: appropriate medication should be provided to the patient according to the doctor's advice based on the disease condition, and the patient should not arbitrarily change drug usage; medication with the dosage based on the blood glucose level should be administered, without any increment or reduction. For example, sulfonylurea drugs should be taken 30 minutes before meals, drugs in the Glinides class should be taken daily before the main meal, biguanide drugs should be taken approximately 30 minutes after meals, and a-glucosidase inhibitors can be swallowed before meals, which must be taken together with the first bite of the meal and must be swallowed after chewing. The gastrointestinal adverse reactions should be carefully observed. For the injection of insulin, the dose must be accurate, and shaking should be avoided when taking it by suction, with strict control of the injection time. The site for subcutaneous injection should be changed in a specific order. (5) Education for patients with DM: four chronic complications may occur in elderly patients with DM. The major vascular complications are obstructive cerebrovascular disease and coronary heart disease. Obstructive cerebrovascular disease is more common as a cerebrovascular disease, whereas coronary heart disease is the most common as a cardiovascular disease; Among the microvascular complications, renal microvascular disease may present as proteinuria, hypertension, and swelling, and renal dysfunction may occur in the late stage; fundus microvascular disease can lead to blindness in diabetic patients. Neurological complications occur as follows: peripheral nerve abnormalities present as pain, numbness, and sensory hypersensitivity; motor nerve disorders can cause localized muscle atrophy; autonomic neuropathy can cause sweating abnormalities, changes in BP and heart rate, urinary incontinence or urinary retention, diarrhea, or constipation.

\subsubsection{Stroke in the elderly}

Stroke, also known as cerebrovascular accident, has the common clinical features of sudden onset and rapid development of limited or diffuse brain dysfunction. Stroke has become the leading cause of death in China. Stroke is also the main cause of disability in the elderly, with $75 \%$ of survivors suffering disability of varying degrees and $40 \%$ suffering severe disability. Stroke includes hemorrhagic stroke and ischemic stroke. Hemorrhagic stroke accounts for approximately $20 \%-30 \%$ of all stroke cases, including cerebral hemorrhage and subarachnoid hemorrhage. Ischemic stroke is a cerebral infarction, accounting for approximately $70 \%-80 \%$ of all stroke cases, including cerebral thrombosis, cerebral embolism, and lacunar infarction.

\subsubsection{Case analysis}

A patient, Mr. Zhu, male, 69 years old, was admitted mainly due to "right limb weakness with alalia for 1 hour". Onset in the patient occurred when he was asleep at night, with cough and vomiting. The patient had a 12-year history of DM, occasional high BP, and a 40-year history of smoking and drinking. The physical examination at admission showed BP of 150/98 mmHg, hemiplegia, hemianopia, aphasia, and muscle strength at level 1.

\subsubsection{Care}

(1) Close observation: changes in the patient's consciousness, pupils, vital signs, and muscle strength should be closely observed to maintain the airway unobstructed. (2) Medication care: aspirin and other platelet aggregation inhibiting drugs can be used in the beginning of the acute phase, but they cannot be used within 24 hours after thrombolysis. Within 3-6 hours after onset, intravenous thrombolytic agents can be used. During thrombolysis, close observation should be performed to carefully prevent intracranial hemorrhage. When mannitol is used to reduce intracranial pressure, the infusion should be fast to avoid extravasation, and the intake and output volume within 24 hours should be recorded. (3) Rehabilitation exercises: these include training in movement ability, coordination ability, and language. In the training, the patient should be respected, and his or her safety should be ensured. (4) Health education: if the symptoms of hernia, such as severe headache, jet vomiting, irritability, increased BP, slowed pulse, deepened disturbance of consciousness, irregular breathing, and bilateral pupils in different sizes, are observed, the doctor should be informed immediately.

\section{Conclusions}

In summary, simultaneously with the continuous improvement in the health conditions of the elderly, there are 
still $18.3 \%$ of the elderly who are in a disabled or partially disabled state, corresponding to a total of 40.63 million people in China. Under normal circumstances, every three disabled elderly individuals need a member of the nursing staff. The demand for elderly care workers in China is approximately 10 million, but the current staff in pension agencies in China amounts to less than 1 million. ${ }^{6}$ Therefore, everyone should have some basic nursing skills for the elderly, which can not only provide services for families but also,

\section{References}

1. Deng KS, Zhong QL. Elderly Nursing. 1st ed. Beijing: China Medical Science and Technology Press; 2016. (in Chinese).

2. Shang SM. Practical Training for Elderly Care Nurses. 1st ed. Beijing: Peking University Medical Publishing House; 2014. (in Chinese).

3. Liu XX. Clinical observation of the efficacy and application of Meipikang dressing in the treatment of inflammatory pressure ulcers in infiltration and its application. J Baotou Med Coll. 2014;30:104-105. (in Chinese). most importantly, improve the quality of life of the elderly and their happiness index. In addition, we also wish to establish a new mode of disability care so that all senior citizens will be looked after properly and can always have something to rely on to enjoy their life in old age.

\section{Conflicts of interest}

All contributing authors declare no conflicts of interest.

4. Dong JW, Mo YZ. Research progress in pain nursing for elderly patients with cancer. J Nurs J. 2016;22:52-54. (in Chinese).

5. Zhan HL. Care of various vessels for elderly patients. In the Proceedings of the 14th National Geriatric Care Academic Conference; 2011: 334-336. (in Chinese).

6. Liang HJ. Study of the Model of Health Care with the Combination of Medicine and Pension for the Elderly in Urban Agencies. Harbin: Heilongjiang Academy of Social Sciences; 2015. (in Chinese).

How to cite this article: Zhu RF, Han SF. Medical care and clinical practice for disabled elderly. Frontiers Nurs. 2018; 1: 07-16. https://doi.org/10.1515/fon-2018-0002 